\title{
Hemiarthroplasty vs. locking plate osteosynthesis - what is the better solution for displaced proximal humerus fractures?
}

Piotr Rusin ${ }^{1}$, Maciej Piotrowski², Tomasz Mazurek ${ }^{2}$, Piotr Lukasiewicz ${ }^{1}$, Adam Nogalski ${ }^{1}$

${ }^{1}$ Department of Trauma Surgery and Emergency Medicine, Medical University of Lublin, Lublin, Poland

2Department of Orthopaedics and Musculoskelatal Traumatology, Medical University of Gdansk, Gdansk, Poland

Submitted: 16 August 2016

Accepted: 4 February 2016

Arch Med Sci Civil Dis 2017; 2: e13-e20

DOI: https://doi.org/10.5114/amscd.2017.66357

Copyright $\odot 2017$ Termedia \& Banach

\section{Abstract}

Introduction: Displaced proximal humerus fractures pose significant clinical problems. The aim of this study was to compare treatment results between open reduction and internal fixation (ORIF) and hemiarthroplasty (HA).

Material and methods: The study was a retrospective assessment. Sixtythree patients (30 HA, 33 ORIF) met the inclusion criteria; mean age: 64.5 years. The Constant-Murley scale, DASH score and VAS surveys for pain and satisfaction were used to evaluate the results.

Results: The HA group: the constant score was 44 points, and the DASH score was 57 points; $53 \%$ of patients had osteolysis of the greater tubercle; none of the patients had revision surgery. ORIF group: the constant score was 59 points, and the DASH score was 38 points; $21 \%$ of patients had a vascular necrosis; revision surgery was performed in $18 \%$ of cases. A significant correlation between good functional outcomes and young age of patients was found in the ORIF group ( $p$-value < 0.048). Patients who started physical therapy earlier achieved better results. DASH scores were better compared to ones from the objective Constant-Murley score. There was no difference in satisfaction between HA and ORIF groups ( $p$-value $<0.1)$.

Conclusions: The ORIF should be considered for patients $<60$ years old, but gives increased risk of urgent revision, due to screw protrusion. The HA provides fewer complications, lower risk of revision and can be better for patients between 60 and 70 years old. When choosing the treatment method, we should avoid focusing only on fracture configuration, but should also consider patient-related factors, such as age and lifestyle.

Key words: proximal humerus fractures, shoulder hemiarthroplasty, open reduction and internal fixation using angle-stable locking plate, shoulder outcome measures.

\section{Introduction}

Proximal humerus fractures represent $4 \%$ to $5 \%$ of fractures among adults and $10 \%$ of fractures occurring above the age of 65 [1]. The classifications that we use are not fully satisfactory as a guideline for modern treatment and the final outcome indication. Predicting the damage to arterial vessels supplying the humeral head is the basis of AO/OTA classification [2]. 11C/AO fractures are intra-articular fractures with heal-

\author{
Corresponding author: \\ Piotr Rusin MD \\ Department of \\ Trauma Surgery and \\ Emergency Medicine \\ Medical University \\ of Lublin \\ 16 Stanisława Staszica St \\ 20-081 Lublin, Poland \\ Phone: +48 505477464 \\ E-mail: piotrrusin@gumed. \\ edu.pl
}


ing results often being disappointing [3]. When analyzing the international bibliography it can be noted that so far no algorithm for therapeutic procedures has been established and that there are no clear guidelines regarding the choice of best method of treatment $[3,4]$. Correct qualification for the procedure and adequate surgical technique are important. There are many controversies regarding whether better results are obtained by internal fixation or hemiarthroplasty of the shoulder joint $[5,6]$. For patients above the age of 70 one solution can be total reverse shoulder replacement, where functional results for this method are promising $[7,8]$. In younger patients the choice of an appropriate method is still a problem that has not been entirely solved.

The aim of this study was to compare between the results of surgical treatment of displaced $11 \mathrm{C} /$ AO fractures using an angle stable locking plate and hemiarthroplasty, and to determine whether there are any variables having an impact on the final treatment results, which method is characterized by more revision surgery, and whether the objective result of the shoulder function (Constant-Murley score) is comparable with the subjective perceptions of a patient (DASH score, VAS satisfaction).

\section{Material and methods}

During the years 2009-2013, 233 patients in the Department of Orthopedics and Trauma Surgery of the Medical University of Gdansk were operated on because of proximal humerus fractures (57 treated using hemiarthroplasty, 176 using plate fixation). Sixteen patients died ( 6 after hemiarthroplasty, 10 after plate fixation) and 61 people were lost from the observation group (follow-up). Thirty-two cases were excluded from the study due to the sustained injury or paresis of the opposite upper limb. From the remaining 124 patients 63 cases were displaced 11C/AO fractures (47 females, 16 males with the observation period longer than 12 months, mean age: 64.5 years $(S D=13.7)$, mean follow-up period 29 months $(S D=13.2)$. Thirty patients were treated using shoulder hemiarthroplasty and 33 patients using an angle stable locking plate. Since the study included patients without a history of any disease of the opposite shoulder joint, it was assumed that the opposite shoulder has $100 \%$ range of motion (constant 40 points) and $100 \%$ of strength for the given patient. Every patient gave informed consent to participate in the clinical trial and to use radiological and photographic documentation in the scientific study. Research permission was granted by the Independent Bioethical Commission for Scientific Research at the Medical University of Gdansk. Fracture types 11C2-C3/
AO were classified based on X-ray examination and, where in doubt, a computed tomography (CT) scan was performed. All the patients had X-rays done in two projections - AP and lateral (transthoracic) or scapular y-view during trauma, after surgery and after the treatment came to an end. Open reduction and internal fixation (ORIF) post-operative $X$-rays were used to evaluate femoral neck-shaft angle, reposition of the articular surface and greater tubercle. Non-anatomical reposition was determined when: fragments of the articular surface were dislocated by $>2 \mathrm{~mm}$, the correct femoral neck-shaft angle of $135^{\circ}$ was decreased or increased by $20^{\circ}$ in the AP projection [9], or the greater tubercle was dislocated by $>10 \mathrm{~mm}$ [10]. On post-operative hemiarthroplasty $X$-rays greater tubercle reposition was evaluated using the Boileau score $[11,12]$. Final radiological assessment was used to evaluate bone union or its disorders, presence of avascular necrosis of the humeral head or greater tubercle, occurrence of inflammatory osteolysis, occurrence of a conflict between the implant and coracoid process of the scapula, disruption of relations in the joint (dislocation, subluxation), heterotopic ossification and complications connected with implants (loosening, protrusion of the locking elements). Choice of a given operative treatment was done both before and intraoperatively (in case where stable osteosynthesis was not possible, conversion to hemiarthroplasty was performed). Procedures were done by three experienced surgeons (P.M., K.S., D.K.).

All the patients were operated on under regional anaesthesia (brachial plexus block) and/or under general anaesthesia. The patient was seated in the "beach chair" position. A deltopectoral approach was used [13]. Philos Synthes implants were used in cases of ORIF. Reposition of tubercles was strengthened by nonabsorbable 2.0 sutures, which were stitched to the plate. Whenever possible, efforts were made to obtain stable fixation of three main fragments, i.e. the head and greater and lesser tubercle, using screws locked in the plate. The next step was fixation of the proximal end of the humerus with the shaft. Hemiarthroplasty was performed using implants from three companies (Epoca Synthes - 25 patients, Aequalis Tornier - 5 patients; choice of an implant was dictated by availability in the centre). After mounting the head of the prosthesis, the stem was fixed within the medullary cavity on bone cement in retrotorsion of about $20^{\circ}$ determined from the long axis of the humerus. The level of endoprosthesis fixation was $5 \mathrm{~cm}$ for men and $4 \mathrm{~cm}$ for women above the level of pectoralis major muscle attachment. The space between the stem and humeral tubercles and the space in the metaphysis were filled with grafts from the resected humeral head. Next, using nonabsorbable 2.0 sutures the greater 
and lesser tubercles were sutured along with rotator cuff attachments $[11,14]$. This was then additionally strengthened with a loop put over the neck and tubercles of the humerus. In patients treated using arthroplasty tenodesis of the biceps brachii long head tendon was performed. The limb was put on a sling after surgery. In case of doubts about stability of the fixation or joint after arthroplasty patients were provided with shoulder stabilizer for 3 to 6 weeks. Upon discharge from hospital all the patients were given a protocol of exercises to perform at home, which included passive exercises of bending the shoulder to the front and abduction until the $3^{\text {rd }}$ week, then for the next 3 weeks additional passive exercises of external and internal rotation and 6 weeks after surgery active exercises. The time for starting rehabilitation under supervision of medical supervision has varied (in Poland the system is based on queues or optionally private). Six weeks after surgery patients had shoulder radiographs done in two projections or more frequently/later done in case of complications. Clinical analysis (minimum 12 months after surgery) was based on pain assessment, range of motion, shoulder muscle strength and satisfaction with the performed treatment. The Constant-Murley scale [15] and DASH score [16] were used for functional assessment of the shoulder. The VAS scale was used to assess pain and satisfaction with treatment. Range of motion was examined using a goniometer. Muscle strength was examined using a spring balance with the limb abducted to $90^{\circ}$ in the shoulder joint, the elbow joint extended and the forearm pronated. If the patient was not able to perform $90^{\circ}$ abduction, measurement was done in the highest achieved position. The best result of strength of the affected limb was the percentage of maximum strength of the healthy limb. The obtained percentage value was divided by 4 to obtain a result on a 0-25 point scale [17].

\section{Statistical analysis}

In the study quantitative traits were described with basic statistics using the mean and standard deviation (SD). For the confidence interval at which the result was considered statistically significant the $p$-value was set as $<0.05$. Student's $t$-test was used to compare two means from independent trials. For correlation analysis Pearson's coefficient for continuous variables was used. Statistical calculations were performed using SPSS software for Windows v 19.

\section{Results}

Constant analysis showed better function of shoulders treated using ORIF. The range of mo- tion was better in the following planes: flexion, abduction, external rotation. There was no difference in the range of external rotation. Strength of shoulders treated using ORIF was also better, but no statistically significant difference was found. DASH score analysis also showed better function of the limb among ORIF treated patients. Comparing the obtained results it was found that the DASH score does not correspond to the results of the more restrictive and objectified Constant-Murley scale. In the group treated using hemiarthroplasty there were almost 4 times more good or very good results in patients' subjective opinion, and the ORIF group showed almost twice as many of such results (Table I). Eighty percent of patients were satisfied with their treatment regardless of the used method, and most of the patients described the level of pain using the Constant scale as mild or no pain (ORIF $82 \%$, hemiarthroplasty $56 \%$ ) (Table II). In $96.7 \%$ of cases (29 out of 30 ) after hemiarthroplasty, fixation of the implant and greater tubercle reposition (dislocation < $10 \mathrm{~mm}$ [10]) were satisfactory in the postoperative images. Sixteen out of 30 cases after hemiarthroplasty showed a complication in the form of delayed union and osteolysis of the greater tubercle, whereas patients who did not present with resorption of the greater tubercle (14 out of 30 cases) had better functional results (constant 60.1 points; DASH 41.8 points). Those results were close to the results of patients treated using ORIF. In our material among patients after hemiarthroplasty heterotrophic ossifications were not very severe and functional results did not differ from the results of the entire group (constant 46.6 points; DASH 53.3 points). None of the patients required urgent surgical intervention in the early postoperative period (12 months). The most common radiological problem visible on postoperative images after ORIF was incomplete anatomical repositioning of the proximal fragment of the humerus $39.3 \%$ of cases). Avascular necrosis of the humeral head or greater tubercle was observed in $21.2 \%$ of cases. If the changes applied to the head fragment, it highly influenced the general result (constant 17.6 points; DASH 76.6 points). Changes applying to the greater tubercle were not progressive and the obtained results were much better (constant 66.4 points; DASH 26 points). In $18.2 \%$ of cases (6 patients) required urgent reoperation due to protrusion of the blocking elements (Table III). In the case of patients treated with plate fixation, young patients showed betters treatment results, which was not observed among patients treated with hemiarthroplasty. Neither of the groups showed dependence of general results on time elapsed between injury and surgery (mean of 10 
Table I. Comparison of results

\begin{tabular}{|c|c|c|c|}
\hline Parameter & HA & ORIF & $P$-value \\
\hline VAS pain score & 4.3 & 2.6 & $<0.02$ \\
\hline Constant pain score & 7.7 & 10.3 & $<0.026$ \\
\hline Flexion $\left[^{\circ}\right]$ & 82 & 109 & $<0.002$ \\
\hline Abduction $\left[{ }^{\circ}\right]$ & 83 & 97 & $<0.032$ \\
\hline External rotation $\left[{ }^{\circ}\right]$ & 17 & 26 & $<0.015$ \\
\hline Internal rotation $\left[{ }^{\circ}\right]$ & 47 & 53 & $<0.291$ \\
\hline Strength $[\mathrm{kg}]$ & 4.6 & 5.8 & $<0.086$ \\
\hline Constant score: & 43.7 & 59 & $<0.009$ \\
\hline Excellent and good results $>70$ points (\%) & 10 & 33.4 & \\
\hline Unsatisfactory results < 56 points (\%) & 76.7 & 39.4 & \\
\hline DASH score: & 57.4 & 38.4 & $<0.004$ \\
\hline Excellent and good results < 51 points (\%) & 36.7 & 63.6 & \\
\hline Unsatisfactory results $>75$ points (\%) & 30 & 9.1 & \\
\hline VAS satisfaction score & 7 & 7.7 & $<0.1$ \\
\hline
\end{tabular}

Table II. Characteristics of groups. Results in terms of variables ( $r$ - Pearson correlation)

\begin{tabular}{|c|c|c|}
\hline Parameter & $\mathrm{HA}(N=30)$ & ORIF $(N=33)$ \\
\hline Sex & 24 female, 6 male & 23 female, 10 male \\
\hline Follow-up [months] & $39.1(\mathrm{SD}=18.5)$ & $29.2(S D=13.5)$ \\
\hline AO C2 (\%) & 30 & 78.8 \\
\hline AO C3 (\%) & 70 & 21.2 \\
\hline Age [years] & $68.9(\mathrm{SD}=11.4)$ & $62.3(S D=14.3)$ \\
\hline$r$ Constant score & $p<0.41$ & $p<0.048$ \\
\hline DASH score & $p<0.97$ & $p<0.60$ \\
\hline Days from injury to surgery & $13.4(\mathrm{SD}=13.9)$ & $8.11(\mathrm{SD}=7.1)$ \\
\hline$r$ Constant score & $p<0.94$ & $p<0.93$ \\
\hline DASH score & $p<0.39$ & $p<0.72$ \\
\hline Start medical rehabilitation [weeks] & $8.3(\mathrm{SD}=3.5)$ & $5.3(\mathrm{SD}=3.6)$ \\
\hline$r$ Constant score & $p<0.032$ & $p<0.007$ \\
\hline DASH score & $p<0.001$ & $p<0.015$ \\
\hline
\end{tabular}

days). In turn, shorter time taken to start postoperative rehabilitation under medical supervision increased the chances for a better final outcome regardless of the chosen method of treatment.

\section{Discussion}

Despite continuously improving systems, treatment of multifragmentary fractures - mainly displaced $11 \mathrm{C} / \mathrm{AO}$ - is still a challenge for surgeons [18]. Those fractures relate mainly to patients about and above the age of 60 , main- ly females with bones affected by osteoporosis, often accompanied by a damaged rotator cuff or arthritic changes to the joint. Precise anatomical realignment of severe dislocated fractures among these patients is still a problem [19]. Due to this, there is still no consensus regarding the method of treatment of such fractures. Performing hemiarthroplasty in the case of such fractures gives relief from pain $[11,17]$, primarily compared to patients treated conservatively [20]. The main cause of pain among patients after hemiarthroplasty 
according to Boileau et al. and Kontakis et al. [11, 12] is impingement syndrome caused by upward subluxation of the prosthesis. This can be seen in our group of patients, where osteolysis of the greater tubercle after shoulder arthroplasty and in consequence upward dislocation of the head were found in the observations. Pain was higher; four patients described it as chronic (Figure 1). In the case of patients treated with fixation Spross et al. [21] report that the protrusion of screws to the joint and irritation of the glenoid cavity cause severe shoulder pain, as in the case of our study. The range of shoulder motion after sustained $11 \mathrm{C} / \mathrm{AO}$ fracture both among those treated with osteosynthesis and those treated with hemiarthroplasty demonstrates limitations in all planes. Robinson et al. report that after surgical treatment of multifragmentary fracture of the proximal part of the humerus patients regain an average of $50 \%$ of shoulder mobility [17], which is supported by the results of our study (ORIF group 58\%, hemiarthroplasty group 42\%). Only in 7 (23.3\%) cases treated using arthroplasty did we observe flexion and abduction exceeding $90^{\circ}$. Comparable results were reported by Kralinger (167 cases of fractures treated with hemiarthroplasty), where abduction to the scapula level is achieved by only $35 \%$ of patients [22]. Most authors report unanimously that shoulder mobility after hemiarthroplasty is not fully satisfactory: Robinson et al. 24/40 points [17], Cai et al. 33.9/40 points [9], while in our study patients scored $17 / 40$ points (Figure 2). It can be concluded that suffering a 11C/ $\mathrm{AO}$ fracture is associated with future limitation in mobility as well as shoulder muscle strength. The literature gives a rate from $21 \%$ to $53 \%$ of problems connected with greater tubercle union
Table III. Complications

\begin{tabular}{|c|c|c|}
\hline \multicolumn{2}{|c|}{ Complications } & \multirow{2}{*}{$\begin{array}{c}\% \\
53.3\end{array}$} \\
\hline HA & Osteolysis of the greater tuberosity & \\
\hline & Impingement syndrome & 60 \\
\hline & Superficial infection & 3.3 \\
\hline & Ectopic calcification & 10 \\
\hline & Revision surgery & 0 \\
\hline \multirow[t]{7}{*}{ ORIF } & Fragments of the articular surface $>2 \mathrm{~mm}$ & 6.1 \\
\hline & Varus deformity & 33.3 \\
\hline & Perioperative protrusion screw & 6.1 \\
\hline & Avascular necrosis & 21.2 \\
\hline & Impingement syndrome & 18.2 \\
\hline & Superficial infection & 6.1 \\
\hline & Revision surgery & 18.2 \\
\hline
\end{tabular}

after hemiarthroplasty (53.3\% in our study) [11, $12,22]$. Lack of union and osteolysis of the greater tubercle are relatively frequent among patients after hemiarthroplasty, which leads to rotator cuff dysfunction and disappointing functional results [23]. Upward migration of the prosthesis is observed and patients are not able to lift the limb to more than $90^{\circ}$. Similar dependence is presented in studies by Bolieau et al. and Kontakis et al. [11, 12]. There are studies with good and very good functional results of shoulders after hemiarthroplasty with greater tubercle union in an anatomical position [11, 24]. Still, the question remains why more than half of correctly aligned greater tubercles underwent osteolysis in our study ma-
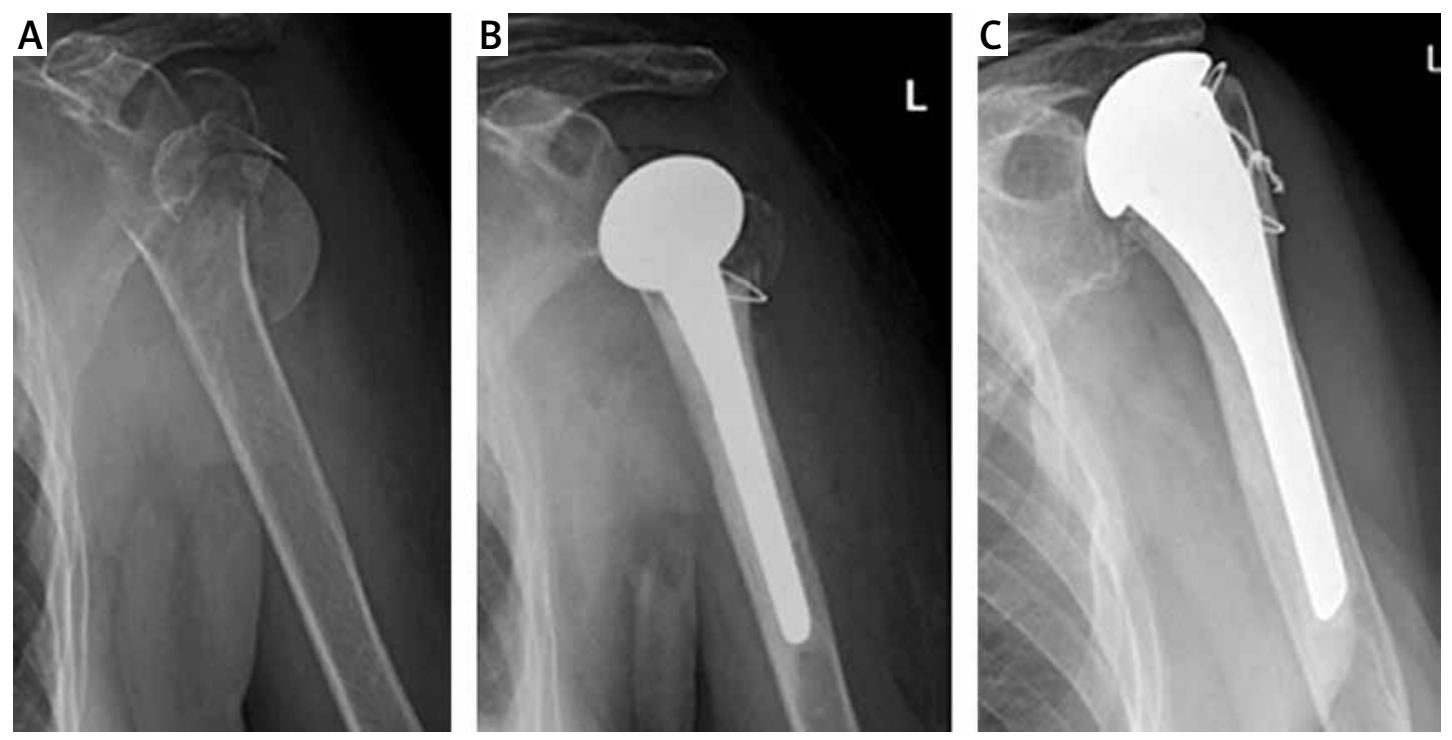

Figure 1. Radiographs obtained at: A - the time of injury, B - postoperatively, C - after 24 months follow-up. The 78-year-old patient (constant 35 points, DASH 88 points) 

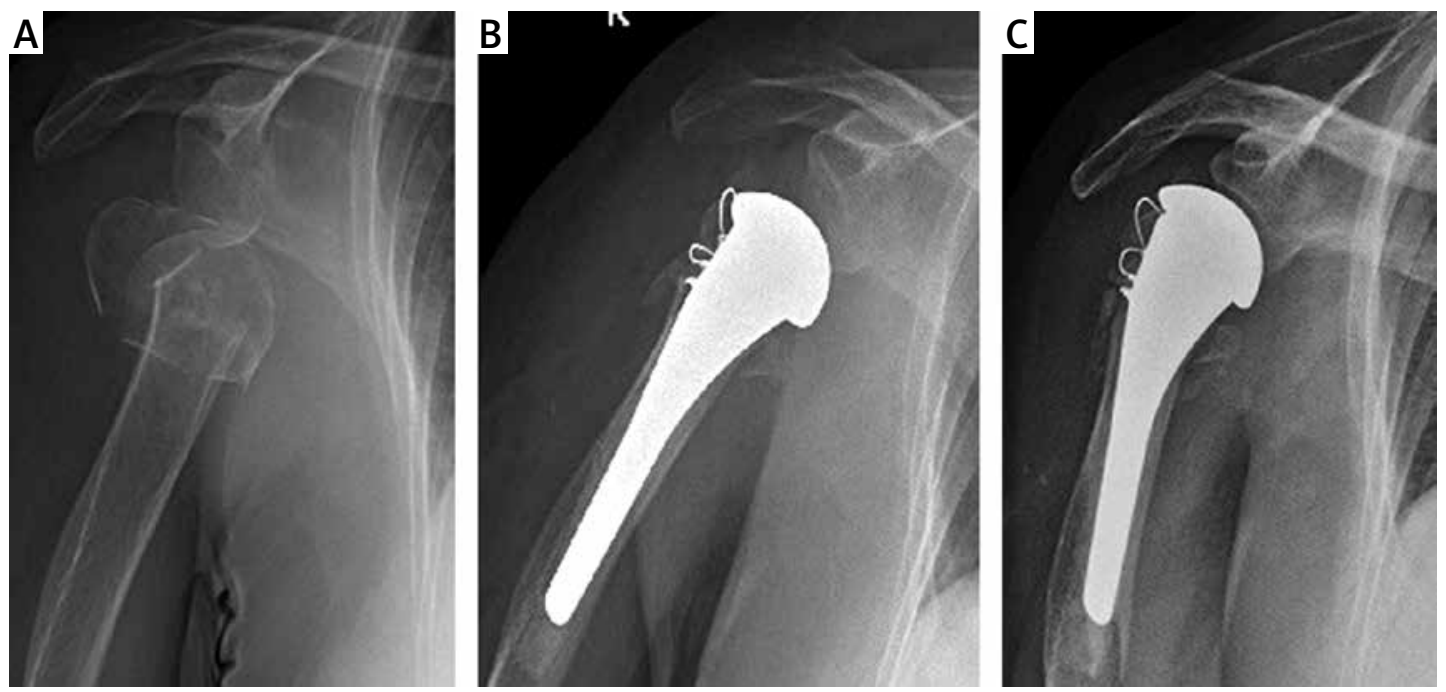

Figure 2. Radiographs obtained at: A - the time of injury, B - postoperatively, C - after 3 months follow-up. The 80 -year-old patient (after 24 months follow-up: constant 58 points, DASH 49 points)

terial. Companies producing implants compete with each other in ideas regarding tubercle fixation, yet no univocal method has been found so far. Perhaps when finding fractures of the greater tubercle on a preoperative radiograph in the case of younger patients reverse shoulder arthroplasty is worth considering? Observations are distant and not extensive, and this requires further studies. In the group treated using fixation 11 cases showed varus deformity complication (the majority of them resulting in varus collapse) (Figures $3-5)$. The results of those patients are comparable for the entire group of patients after hemiarthroplasty (constant 46.7 points; DASH 47.5 points). Unfortunately, in four patients full necrosis of the humeral head was observed and two others had necrosis of the greater tubercle. Gardner in his work used an allograft from the fibula, using which he reconstructs the medial column, does plate fixation and claims that he does not observe complications such as varus collapse [25]. In our clinic we have not used this solution so far, but I find this method worthy of greater attention. Brunner et al. report that a complication in the form of protrusion of the fixating materials in the direct postoperative period is the main reason for revision in the early period. In their studies they reported protrusion of the locking elements in $14 \%$ of cases (22 out of 157 patients) after plate osteosynthesis [26]. Most commonly this complication is connected with the surgical technique, that is placing the locking screw too close to the articular surface. In the case of patients treated using plate osteosynthesis revision procedures were more frequent, representing 18.2\% (6 out of 33 cases). In comparison to patients treated with hemiarthroplasty, no such cases were reported. Most publications state that ORIF more frequently requires revisions [23]. In their study, Spross et al. [21] qualified patients with type $\mathrm{VI}$ Neer fracture for comparative analysis and made a record of $45.5 \%$ of revision procedures in the group of patients treated using plate osteosynthesis (10 out of 22 cases) compared with $4.5 \%$ of procedures in the group of patients after hemiarthroplasty (1 out of 22 cases). Spross et al. [21] did not find better functional results in any of the groups. Cai et al. qualified patients with four-part
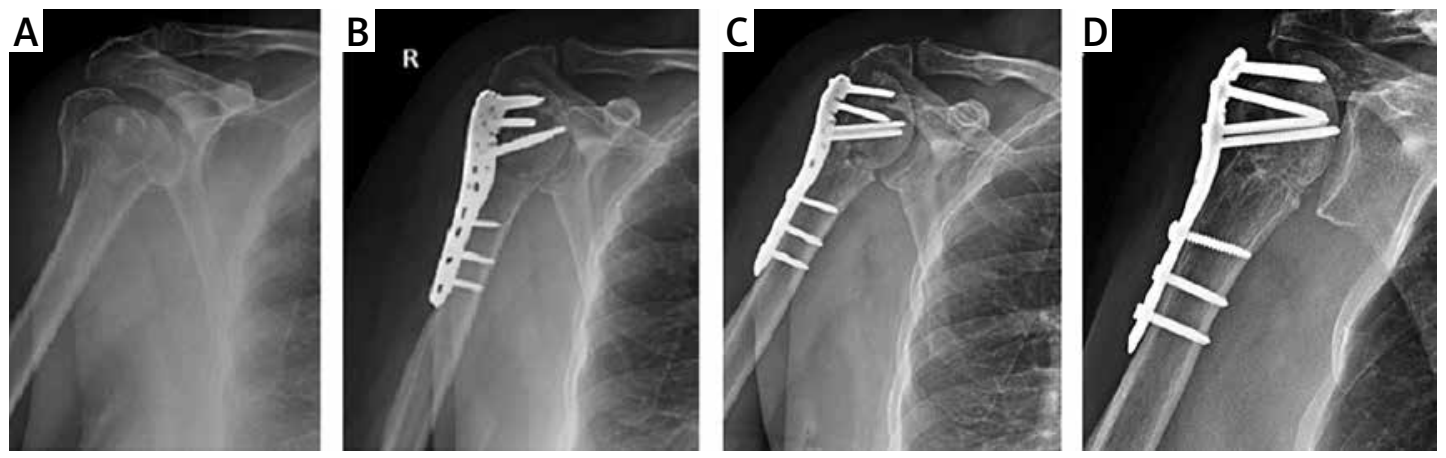

Figure 3. Radiographs obtained at: A - the time of injury, B - postoperatively (varus deformity), C - after 3 months (beginning AVN), D - after 24 months with AVN and consecutive screw cut-out. The 67-year-old patient (constant 34 points, DASH 71 points) 

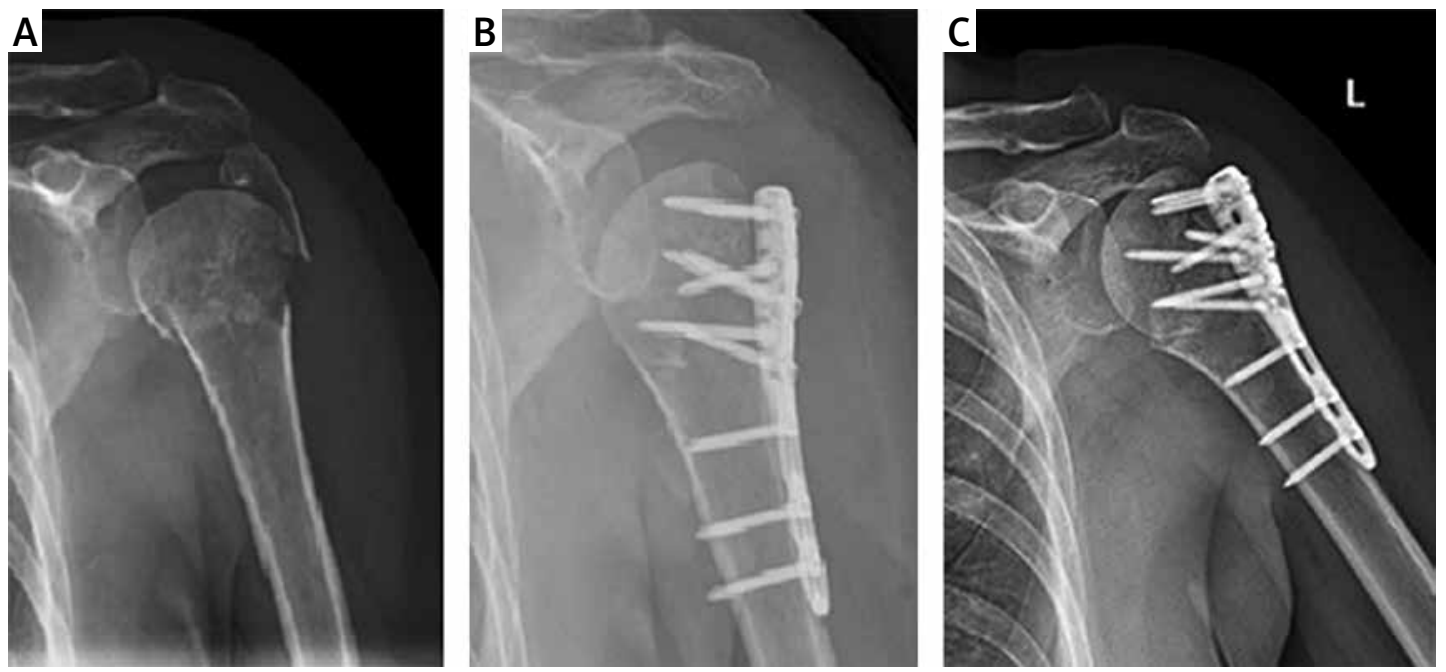

Figure 4. Radiographs obtained at: A - the time of injury, B - postoperatively (varus deformity), C - after 3 months follow-up with partial AVN. The 64-year-old patient (after 24 months of excellent function: constant 94 points, DASH 4 points)
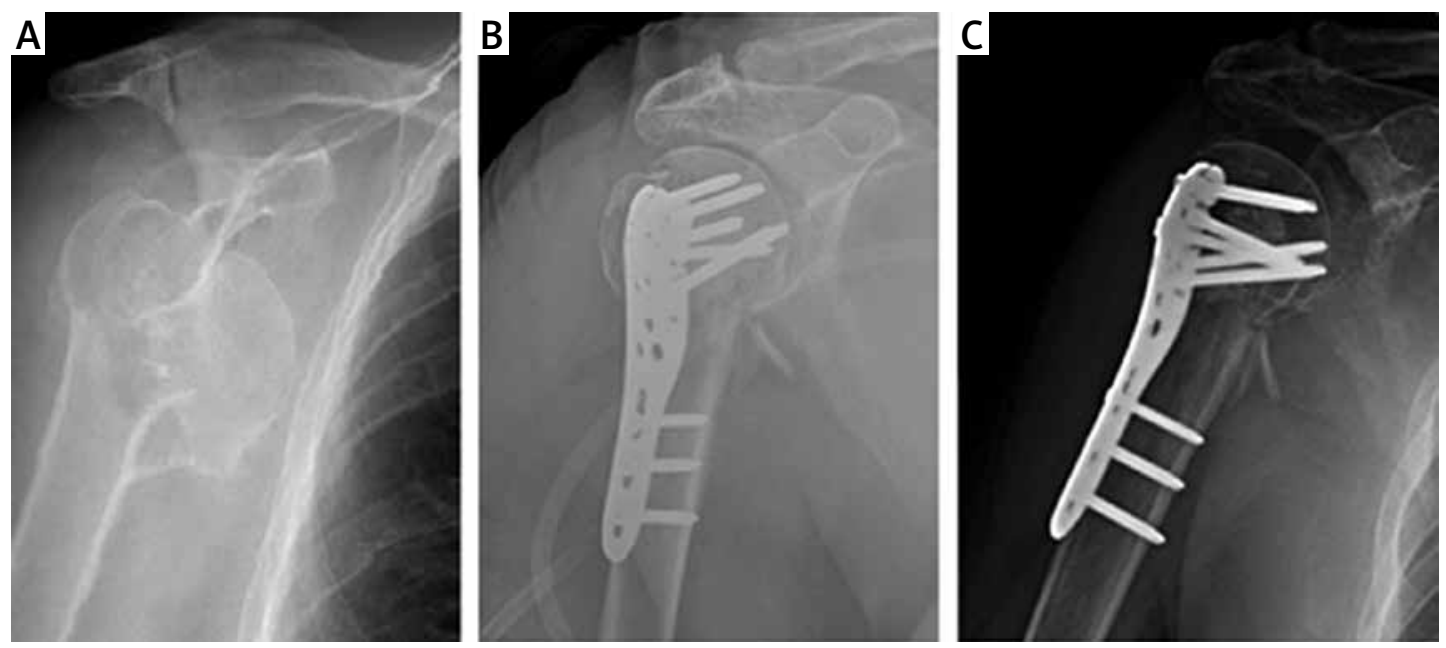

Figure 5. Radiographs obtained at: A - the time of injury, B - postoperatively (varus deformity), C - after 3 months follow-up. The 76-year-old patient (after 24 months of satisfactory function: constant 56 points, DASH 13 points

fractures according to the Neer classification for comparative analysis and found a slight advantage of patients after hemiarthroplasty compared to patients treated using ORIF (the differences were, however, small) [9]. Evaluation of treatment of three-part and four-part fractures according to the Neer classification was performed by Solberg et al. [27]. They reported, as in our study, better functional results in patients treated using osteosynthesis. The best results for shoulder function after hemiarthroplasty in trauma patients were presented by Charles Neer in 1970 [13]. Not many authors have been able to confirm these revelations. Murray et al. reported worse functional results for older patients who are not able to be actively involved in rehabilitation after surgery. In their opinion postponing rehabilitation treatment leads to periarticular adhesions and contractions [23]. In general, the literature does not give a clear answer on which method of treatment is best [4]. Analyzing data obtained from the studies it can be concluded that the functional results do not fully correspond with the treatment satisfaction level. This is supported by patients after hemiarthroplasty who achieved much poorer results in range of motion and shoulder strength, but $80 \%$ of these patients were happy with their treatment.

In conclusion, none of the compared methods of fracture treatment releases patients from pain entirely or restores full mobility of the shoulder joint. In younger patients ( $<60$ years old) ORIF should be considered as a treatment method of choice, as the shoulder range of motion after hemiarthroplasty is very often unsatisfactory. However, when choosing ORIF varus deformity should be avoided due to the high risk of avascular necrosis of the humeral head, screw protrusion to the joint and in consequence urgent revision 
surgery. When preparing for the surgery, conversion from fixation to arthroplasty always needs to be considered with implants ready for both procedures. Despite ORIF giving a better range of motion, it shows higher risk of complications leading to surgical intervention. Hemiarthroplasty is characterized by a low number of complications requiring urgent revision procedures and seems to be a better choice of treatment for patients aged between 60 and 70 years. It is worth mentioning that the satisfaction level among patients treated with hemiarthroplasty and ORIF did not differ. The decision of a given method of treatment should be made during direct conversation between the surgeon and the patient after individual case consideration. A multidisciplinary approach and inclusion of professional rehabilitation treatment are also important.

\section{Conflict of interest}

The authors declare no conflict of interest.

\section{References}

1. Court-Brown CM, Caesar B. Epidemiology of adult fractures: a review. Injury 2006; 37: 691-7.

2. AO/OTA Fracture Classification AO surgery. https:// www2.aofoundation.org/wps/portal/surgery. Accessed 17 Apr 2015.

3. Slobogean GP, Johal H, Lefaivre KA, et al. A scoping review of the proximal humerus fracture literature. BMC Musculoskelet Disord 2015; 16: 112.

4. Handford C, Nathoo S, Porter K, Kalogrianitis S. A review of current concepts in the management of proximal humerus fractures. Trauma 2014; 17: 181-90.

5. Misra A, Kapur R, Maffulli N. Complex proximal humeral fractures in adults: a systematic review of management. Injury 2001; 32: 363-72.

6. Zyto K, Kronberg M, Broström LA. Shoulder function after displaced fractures of the proximal humerus. J Shoulder Elbow Surg 1995; 4: 331-6.

7. Baudi P, Campochiaro G, Serafini F, et al. Hemiarthroplasty versus reverse shoulder arthroplasty: comparative study of functional and radiological outcomes in the treatment of acute proximal humerus fracture. Musculoskelet Surg 2014; 98: S19-25.

8. Chalmers PN, Slikker W, Mall NA, et al. Reverse total shoulder arthroplasty for acute proximal humeral fracture: comparison to open reduction-internal fixation and hemiarthroplasty. J Shoulder Elb Surg 2014; 23 : 197-204.

9. Cai M, Tao K, Yang C, Li S. Internal fixation versus shoulder hemiarthroplasty for displaced 4-part proximal humeral fractures in elderly patients. Orthopedics 2012; 35: e1340-6.

10. Bahrs C, Oehm J, Rolauffs B, et al. T-plate osteosynthesis - an obsolete osteosynthesis procedure for proximal humeral fractures? Middle term clinical and radiological results. Z Orthop Ihre Grenzgeb 2007; 145: 186-94.

11. Boileau P, Krishnan SG, Tinsi L, et al. Tuberosity malposition and migration: reasons for poor outcomes after hemiarthroplasty for displaced fractures of the proximal humerus. J Shoulder Elb Surg 2002; 11: 401-12.
12. Kontakis G, Koutras C, Tosounidis T, Giannoudis P. Early management of proximal humeral fractures with hemiarthroplasty: a systematic review. J Bone Joint Surg Br 2008; 90: 1407-13.

13. Neer CS $2^{\text {nd }}$. Displaced proximal humeral fractures. II. Treatment of three-part and four-part displacement. J Bone Joint Surg Am 1970; 52: 1090-103.

14. Boileau P, Trojani C, Walch G, et al. Shoulder arthroplasty for the treatment of the sequelae of fractures of the proximal humerus. J Shoulder Elb Surg 2001; 10: 299-308.

15. Constant CR, Murley AH. A clinical method of functional assessment of the shoulder. Clin Orthop Relat Res 1987; 214: 160-4.

16. DASH (Disabilities of the Arm, Shoulder and Hand). http://www.dash.iwh.on.ca/. Accessed 23 Apr 2015

17. Robinson CM, Page RS, Hill RMF, et al. Primary hemiarthroplasty for treatment of proximal humeral fractures. J Bone Joint Surg Am 2003; 85: 1215-23.

18. Pinkas D, Wanich TS, DePalma AA, Gruson KI. Management of malunion of the proximal humerus: current concepts. J Am Acad Orthop Surg 2014; 22: 491-502.

19. Fjalestad T, Strømsøe K, Hole $M \emptyset$, et al. Surgical treatment with an angular stable plate for complex displaced proximal humeral fractures in elderly patients: a randomized controlled trial. J Orthop Trauma 2012; 26: 98-106.

20. Olerud P, Ahrengart L, Ponzer S, et al. Hemiarthroplasty versus nonoperative treatment of displaced 4-part proximal humeral fractures in elderly patients: a randomized controlled trial. J Shoulder Elb Surg 2011; 20: 1025-33.

21. Spross C, Platz A, Erschbamer M, et al. Surgical treatment of neer group vi proximal humeral fractures: retrospective comparison of PHILOS ${ }^{\circ}$ and hemiarthroplasty. Clin Orthop Relat Res 2012; 470: 2035-42.

22. Kralinger F, Schwaiger R, Wambacher M, et al. Outcome after primary hemiarthroplasty for fracture of the head of the humerus. A retrospective multicentre study of 167 patients. J Bone Joint Surg Br 2004; 86: 217-9.

23. Murray IR, Amin AK, White TO, Robinson CM. Proximal humeral fractures: current concepts in classification, treatment and outcomes. J Bone Joint Surg Br 2011; 93: 1-11.

24. Reuther F, Wahl D, Mühlhäusler B, Nijs S. Functional outcome of shoulder hemiarthroplasty for fractures: a multicentre analysis. Injury 2010; 41: 606-12.

25. Gardner MJ, Boraiah S, Helfet DL, Lorich DG. Indirect medial reduction and strut support of proximal humerus fractures using an endosteal implant. J Orthop Trauma 2008; 22: 195-200.

26. Brunner F, Sommer C, Bahrs C, et al. Open reduction and internal fixation of proximal humerus fractures using a proximal humeral locked plate: a prospective multicenter analysis. J Orthop Trauma 2009; 23: 163-72.

27. Solberg BD, Moon CN, Franco DP, Paiement GD. Surgical treatment of three and four-part proximal humeral fractures. J Bone Joint Surg Am 2009; 91: 1689-97. 